\title{
Impact of age at type 2 diabetes mellitus diagnosis on mortality and vascular complications: systematic review and meta-analyses
}

\author{
Natalie Nanayakkara ${ }^{1,2}$ (D) - Andrea J. Curtis ${ }^{1}$ (D) $\cdot$ Stephane Heritier $^{1}$ (D) - Adelle M. Gadowski ${ }^{1}$ (D) Meda E. Pavkov $^{3}$ (D) \\ Timothy Kenealy ${ }^{4}$ (D) - David R. Owens ${ }^{5}$ (D) - Rebecca L. Thomas ${ }^{5}$ (D) - Soon Song ${ }^{6}$ (D) - Jencia Wong ${ }^{7}$ (I) - Juliana C.- \\ N. Chan $^{8}$ (D) Andrea O.-Y. Luk ${ }^{8}$ (D) - Giuseppe Penno ${ }^{9}$ (D) Linong Ji ${ }^{10}$ (D) Viswanathan Mohan $^{11}$ (D) \\ Anandakumar Amutha ${ }^{11}$ (D) - Pedro Romero-Aroca ${ }^{12}$ (D) - Danijela Gasevic ${ }^{1,13}$ (D) - Dianna J. Magliano ${ }^{1,2}$ (D) \\ Helena J. Teede ${ }^{14}$ (D) John Chalmers ${ }^{15}$ (D) Sophia Zoungas ${ }^{1,15}$ (iD
}

Received: 8 May 2020 / Accepted: 2 September 2020 / Published online: 14 December 2020

(C) The Author(s) 2020

\begin{abstract}
Aims/hypothesis Few studies examine the association between age at diagnosis and subsequent complications from type 2 diabetes. This paper aims to summarise the risk of mortality, macrovascular complications and microvascular complications associated with age at diagnosis of type 2 diabetes.

Methods Data were sourced from MEDLINE and All EBM (Evidence Based Medicine) databases from inception to July 2018. Observational studies, investigating the effect of age at diabetes diagnosis on macrovascular and microvascular diabetes complications in adults with type 2 diabetes were selected according to pre-specified criteria. Two investigators independently extracted data and evaluated all studies. If data were not reported in a comparable format, data were obtained from authors, presented as minimally adjusted ORs (and 95\% CIs) per 1 year increase in age at diabetes diagnosis, adjusted for current age for each outcome of interest. The study protocol was recorded with PROSPERO International Prospective Register of Systematic Reviews (CRD42016043593).

Results Data from 26 observational studies comprising 1,325,493 individuals from 30 countries were included. Random-effects meta-analyses with inverse variance weighting were used to obtain the pooled ORs. Age at diabetes diagnosis was inversely associated with risk of all-cause mortality and macrovascular and microvascular disease (all $p<0.001)$. Each 1 year increase in
\end{abstract}

Sophia Zoungas

sophia.zoungas@monash.edu

Danijela Gasevic

danijela.gasevic@monash.edu

1 School Public Health and Preventive Medicine, Monash University, Melbourne, VIC, Australia

2 Baker Heart and Diabetes Institute, Melbourne, VIC, Australia

3 Centers for Disease Control and Prevention, Division for Diabetes Translation, Atlanta, GA, USA

4 Department of Medicine, University of Auckland, Auckland, New Zealand

5 Diabetes Research Group, Swansea University Medical School, Swansea, Wales, UK

6 Department of Diabetes, Northern General Hospital, Sheffield, UK

7 Royal Prince Alfred Hospital, Camperdown, NSW, Australia
8 Department of Medicine and Therapeutics, The Chinese University of Hong Kong, Hong Kong Special Administrative Region, Shenzhen, People's Republic of China

9 Diabetes and Metabolic Disease Section, Department of Clinical and Experimental Medicine, Azienda Ospedaliero-Universitaria Pisana University of Pisa, Pisa, Italy

10 Department of Endocrinology, Peking University People's Hospital, Xicheng District, Beijing, China

11 Madras Diabetes Research Foundation \& Dr Mohan's Diabetes Specialities Centre, Chennai, India

12 Hospital Universtario Sant Joan, Reus, Spain

13 Usher Institute, University of Edinburgh, Old Medical School, Teviot Place, Edinburgh, UK

14 Monash Centre for Health Research and Implementation, Monash University, Clayton, VIC, Australia

15 The George Institute for Global Health, Camperdown, NSW, Australia 


\section{Research in context}

\section{What is already known about this subject?}

- Type 2 diabetes is increasingly diagnosed at a younger age

- The pathogenesis of the long-term vascular complications associated with early-or late-onset type 2 diabetes is not well characterised

- Studies examining the relationship between age at diagnosis of type 2 diabetes and long-term complications vary widely in population characteristics or methodological rigour and report inconsistent findings (younger age at diabetes diagnosis is associated with increased risk of complications, decreased risk of complications, no difference in risk of complications or variable effects in different end organs)

\section{What is the key question?}

- What is the risk of mortality, macrovascular complications and microvascular complications associated with age at diagnosis of type 2 diabetes mellitus?

What are the new findings?

- This analysis integrates data from over a million people with diabetes worldwide to evaluate the risk of a range of diabetes complications with respect to age at diagnosis

- Each 1 year increase in age at diabetes diagnosis was associated with a $4 \%, 3 \%$ and $5 \%$ decreased risk of all-cause mortality, macrovascular disease and microvascular disease, respectively

\section{How might this impact on clinical practice in the foreseeable future?}

- Identification and quantification of the higher risk of mortality and vascular disease conferred by younger age at type 2 diabetes diagnosis may enable risk stratification of people early in the condition, providing greater opportunities for interventions to reduce risk of adverse outcomes

age at diabetes diagnosis was associated with a 4\%,3\% and 5\% decreased risk of all-cause mortality, macrovascular disease and microvascular disease, respectively, adjusted for current age. The effects were consistent for the individual components of the composite outcomes (all $p<0.001)$.

Conclusions/interpretation Younger, rather than older, age at diabetes diagnosis was associated with higher risk of mortality and vascular disease. Early and sustained interventions to delay type 2 diabetes onset and improve blood glucose levels and cardiovascular risk profiles of those already diagnosed are essential to reduce morbidity and mortality.

Keywords Age factors - Age of onset - Diabetes · Diabetes complications · Diabetes mellitus, type 2 - Disease progression · Meta-analysis $\cdot$ Prognosis $\cdot$ Systematic review

\section{Introduction}

The IDF estimates that the prevalence of diabetes will rise from 425 million people worldwide in 2017 , to 629 million by 2045 [1]. Type 2 diabetes, conventionally considered a disease of middle and older age, is increasingly diagnosed at a younger age $[1,2]$. Type 2 diabetes and its associated complications contribute to $8.4 \%$ of deaths worldwide, consuming significant healthcare resources [3]; this is likely to rise exponentially given the increasing prevalence of the condition [1].

Despite significant diagnostic, monitoring and treatment advances, type 2 diabetes remains associated with increased mortality and morbidity compared with the general population
[4]. However, the pathogenesis of the long-term vascular complications associated with early- or late-onset type 2 diabetes is not well characterised and although the mechanisms for the development of complications may be similar [5], recent evidence suggests an accelerated course in people diagnosed with early-onset type 2 diabetes [6, 7]. Proposed mechanisms include a longer lifetime exposure to the adverse diabetic milieu and/or early-onset type 2 diabetes representing an inherently more aggressive metabolic phenotype with rapid onset of beta cell failure and insulin resistance compared with late-onset disease [2, 8, 9]. Novel cluster analyses raise the possibility of type 2 diabetes representing a clustering of up to five disease subgroups with distinct age at diagnosis, genetics, mechanisms of disease progression and risk of diabetic 
complications [10]. Of the five groups identified, the 'mild age-related diabetes' subgroup contains elderly people who experience the most benign disease course compared with the 'mild obesity-related diabetes' group, characterised by younger age at onset and obesity.

Several studies have examined the relationship between age at diabetes diagnosis and long-term complications among people with type 2 diabetes. These studies vary widely in population characteristics and methodological rigour and report inconsistent findings (e.g. younger age at diabetes diagnosis is associated with increased risk of complications $[6,7$, 11-15], decreased risk of complications [16, 17], no difference in risk of complications [18] or variable effects in different end organs $[19,20])$. Additionally, some studies have proposed that longer diabetes duration [21, 22] or more adverse cardiovascular risk profiles [23, 24] underlie the greater risk of development of vascular complications associated with type 2 diabetes diagnosed at a younger age, while other studies have suggested that impact of age at diagnosis may vary with ethnicity [25].

Evidence of a clinically meaningful effect of age at diagnosis of type 2 diabetes beyond the ageing process itself would have substantial implications for diabetes prevention and treatment as well as the development and implementation of cardiovascular risk prediction tools. The aim of our study was thus to examine the effect of age at diagnosis of type 2 diabetes on risks of complications, focusing on all-cause mortality, macrovascular events and microvascular events.

\section{Methods}

\section{Data sources and searches}

A systematic search of published literature was conducted in MEDLINE and All EBM (Evidence Based Medicine; www. ovid.com/product-details.904.html) databases (including Cochrane Database of Systematic Reviews, ACP Journal Club Database of Abstracts of Reviews of Effects, Cochrane Central Register of Controlled Trials, Cochrane Methodology Register, Health Technology Assessment and NHS Economic Evaluation Database) using the subject headings and key terms detailed in electronic supplementary materials (ESM) Methods. The study methods and reporting follow the Metaanalyses Of Observational Studies in Epidemiology (MOOSE) and Preferred Reporting Items for Systematic review and Meta-Analysis (PRISMA) guidelines [26, 27]. The study protocol was recorded with PROSPERO international prospective register of systematic reviews (CRD42016043593). The search was limited to humans and English language articles and was initially conducted in July 2016 with no time restrictions and updated in July 2018.

\section{Study selection}

The inclusion criteria were determined a priori (ESM Table 1). To be included, studies had to meet the following criteria: be a study of adult participants with type 2 diabetes investigating the effect of age at diabetes diagnosis on macrovascular and microvascular diabetes complications; the study had to assess one or more of the outcome variables all-cause mortality, macrovascular disease, microvascular disease, retinopathy, nephropathy, neuropathy, CVD, cerebrovascular disease and peripheral vascular disease; the study had to have an available mortality/complication rate, where mortality was either a prespecified primary or secondary outcome, or the methods indicated complete follow-up of participants.

Two independent authors (NN and AMG) assessed the title and abstracts of retrieved records for relevance and duplication. Authors then reviewed the full text of potentially eligible citations to identify studies that fulfilled the inclusion criteria. Any uncertainties regarding study inclusion and data extraction were discussed with an experienced systematic reviewer (AJC), statistician (SH) and senior clinician (SZ). The references cited in the retrieved publications were screened for potentially eligible studies. When several articles from the same study had reported on the same endpoint, only the data representing the longest follow-up were extracted.

\section{Data extraction and quality assessment}

Data were extracted from included studies using a specially developed data extraction form. Information was obtained regarding study design and location, participant characteristics, outcome variables and results. Given the wide variation in data reporting and adjustment for confounders, meaningful interpretation, comparison and meta-analysis was not possible. Therefore, we contacted authors to re-analyse and present data in a homogeneous format to enable data pooling and comparison. Corresponding authors were contacted by email at least twice (if data were not reported in a suitable format) to request data, presented as minimally adjusted ORs $(95 \% \mathrm{CI})$ per 1 year increase in age at diabetes diagnosis, with adjustment for current age (or diabetes duration) for each outcome of interest. This format was chosen as most of the studies presented the results in this way.

Risk of bias of included studies was assessed using a specially developed data extraction form, based on the Newcastle-Ottawa scaling for non-randomised studies [28, 29]. Quality assessment criteria included representativeness of participants, validity of the diagnostic criteria, determination of age at diagnosis, outcome assessment, withdrawals and losses to follow-up. Each study was then allocated a risk of bias rating (ESM Tables 2, 3). 


\section{Exposures}

Current age was reported by each study as age at entry into the study or age at baseline assessment. Age at type 2 diabetes diagnosis was reported as the age of the people at the diagnosis of type 2 diabetes, with diabetes duration reported or calculated as current age minus age at diabetes diagnosis.

\section{Outcomes}

The primary a priori outcomes were all-cause mortality, macrovascular disease (composite of CHD, cerebrovascular disease and peripheral vascular disease) or microvascular disease (composite of retinopathy, nephropathy and neuropathy). The secondary a priori outcomes were retinopathy, nephropathy, neuropathy, CHD, cerebrovascular disease and peripheral vascular disease.

\section{Statistical analysis}

Interdependence of current age, age at diabetes diagnosis and diabetes duration precluded use of all three variables in the same model; hence the use of models containing either age at diabetes diagnosis adjusted for current age or age at diabetes diagnosis adjusted for diabetes duration. Adjustment for current age was to remove the effect of ageing per se. Adjustment for diabetes duration was to remove the effect of the time point at which observations happened to be made in the course of illness for each individual; an individual observed early in their illness would appear to have a longer time to develop complications than the same individual observed late in their illness. For studies reporting in multiple models, we extracted data for both minimally adjusted and maximally adjusted increased risk estimates. Unless otherwise stated, the least adjusted risk estimates from each study were used, provided diabetes duration was included. Review Manager (RevMan) software Version 5.3 was used for all statistical analyses [30].

Data were combined in meta-analyses to calculate pooled risk estimates presented as OR $(95 \% \mathrm{CI})$ of the effect of age at diabetes diagnosis (per year) adjusted for current age (or diabetes duration [supplemental analyses]), on outcomes using both fixed and random-effects models (generic inverse variance method) [31]. There were no significant differences between fixed- and random-effects analyses. Random-effects models are presented given heterogeneity among the studies [32]. Crude data were included where possible, given variable control for confounding factors. However, some articles presented adjusted ORs only.

$I^{2}$ was used to assess heterogeneity with values of $25 \%$, $50 \%$ and $75 \%$ considered low, moderate and high, respectively [33]. Funnel plots were used to explore potential publication bias $[34,35]$. A scatter plot of the $t$ statistic associated with each study-estimate value assessed the contribution of each study to the study-estimate random effect vs the $\log _{10}$ of the SE of the effect.

\section{Results}

\section{Characteristics of included studies}

Electronic database and reference searching yielded 2219 publications, of which 156 were reviewed in full text (Fig. 1). Of 33 eligible studies, 26 studies comprising 1,325,493 individuals were included and seven were excluded because data were not provided in the required format in the publication and attempts to contact authors were not successful. The 26 included studies were either cross-sectional (13 studies) or cohort (13 studies) in design. The updated search in 2018 enabled the inclusion of data from three studies. The mean age of study participants ranged from 21.6 years to 67.4 years. The proportion of female study participants ranged from $42.5 \%$ to $68.6 \%$. Table 1 summarises the characteristics of the included studies, which comprise 1,325,493 participants from 30 countries worldwide.

\section{Primary outcomes}

Effects of age at diabetes diagnosis adjusted for current age on all-cause mortality, macrovascular disease and microvascular disease For all-cause mortality, data from five studies [20, 21, 25, 36, 37], comprising 1,325,493 participants, indicated that each 1 year increase in age at diabetes diagnosis was associated with a $4 \%$ decreased risk of all-cause mortality (OR 0.96 [0.94, 0.99], $p<0.001)$ when adjusted for current age. For macrovascular disease, data from eight studies [20, 23-25, 37-40], comprising 566011 participants, indicated that each 1 year increase in age at diabetes diagnosis was associated with a $3 \%$ decreased risk of macrovascular disease (OR 0.97 $[0.96,0.98], p<0.001)$ when adjusted for current age. For microvascular disease, data from eight studies [20, 24, 25, 38-42], comprising 149,110 participants, indicated that each 1 year increase in age at diabetes diagnosis was associated with a 5\% decreased risk of microvascular disease (OR 0.95 $[0.94,0.96], p<0.001)$ when adjusted for current age. Significant heterogeneity in the magnitude of the effects was evident between studies for these outcomes (all $\chi^{2} p<0.001$, all $I^{2} \geq 93 \%$ ) (Fig. 2).

\section{Secondary outcomes}

Effects of age at diabetes diagnosis adjusted for current age on CHD, cerebrovascular disease, peripheral vascular disease, retinopathy, nephropathy and neuropathy Data for individual vascular complications were available from 13 studies, 
Fig. 1 Flow diagram of study selection process

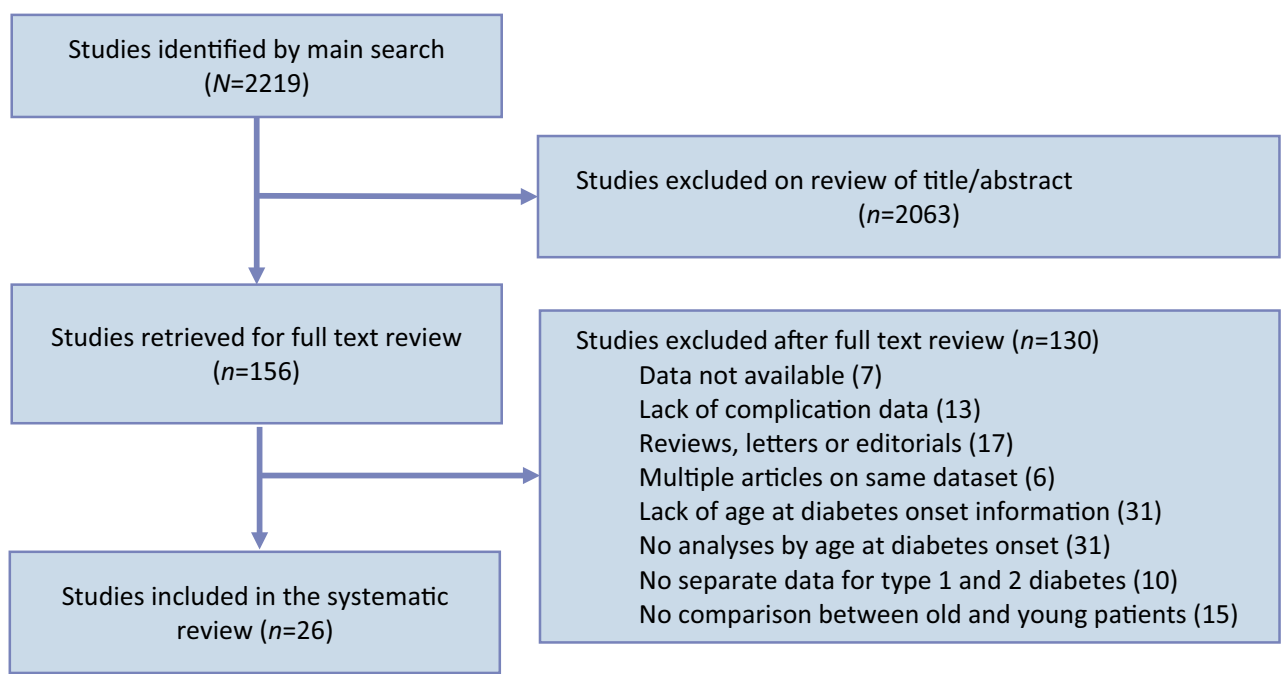

comprising 566011 participants and adjusted for current age. Each 1 year increase in age at diabetes diagnosis was associated with a $2 \%$ decreased risk of CHD (OR 0.98 [95\% CI $0.97,0.98], p<0.001$ ), a $2 \%$ decreased risk of cerebrovascular disease (OR 0.98 [95\% CI 0.97, 0.99], $p<0.001$ ) and a 3\% decreased risk of peripheral vascular disease (OR 0.97 [95\% CI 0.96, 0.99], $p<0.001$ ) (Fig. 3). Each 1 year increase in age at diabetes diagnosis was associated with an $8 \%$ decreased risk of retinopathy (OR 0.92 [95\% CI 0.90, 0.95], $p<0.001$ ), a $6 \%$ decreased risk of nephropathy (OR 0.94 $[0.92,0.96], p<0.001)$ and a $5 \%$ decreased risk of neuropathy (OR 0.95 [95\% CI 0.94, 0.96], $p<0.001$ ) (Fig. 3). Significant heterogeneity in the magnitude of the effects was evident between studies for these outcomes (all $\chi^{2} p<0.001$, all $I^{2} \geq 48 \%$ ).

\section{Sensitivity analyses}

Effects of age at diabetes diagnosis adjusted for diabetes duration on all-cause mortality, macrovascular disease and microvascular disease Data for these analyses were obtained from ten studies comprising 390,139 participants and adjusted for diabetes duration (ESM Fig. 1). Each 1 year increase in age at diabetes diagnosis was associated with a $6 \%$ increased risk of all-cause mortality (OR 1.06 [95\% CI 1.03, 1.09], $p<0.001$ ), a $6 \%$ increased risk of macrovascular disease (OR 1.06 [95\% CI $1.04,1.07], p<0.001)$ and a $5 \%$ increased risk of microvascular disease (OR 1.05 [95\% CI 1.02, 1.08], $p<0.001$ ).

\section{Methodological quality}

Risk of bias assessment of the included studies is presented in ESM Table 3. Study participants were recruited to randomised clinical trials or selected from large clinical datasets. Inclusion and exclusion criteria were adequately described in all studies. Of the included studies, 24 [14, 15, 20, 21, 23-25, 36-52] were of high quality and two $[53,54]$ of medium quality due to insufficient adjustment of confounding variables. In addition, 24 studies [14, 15, 20, 21, 23-25, 36-52] demonstrated low risk of bias and two $[53,54]$ demonstrated a moderate risk of bias due to insufficient adjustment for confounding variables (confounding bias) (ESM Table 3). Funnel plots did not suggest the presence of publication bias (ESM Fig. 2).

\section{Discussion}

This comprehensive systematic review and meta-analysis compiles the results of 26 studies investigating the effects of age at diabetes diagnosis on mortality and subsequent complications in 1,325,493 participants with type 2 diabetes from diverse populations across the Asia Pacific, Europe and North America. We report an inverse relationship between age at diabetes diagnosis and risk of major diabetes complications after adjustment for current age. Each 1 year increase in age at diabetes diagnosis was associated with a $4 \%, 3 \%$ and $5 \%$ decreased risk of all-cause mortality, macrovascular disease and microvascular disease, respectively. These effects were consistent across the individual components of the composite outcomes (CHD, cerebrovascular disease, peripheral vascular disease, retinopathy, nephropathy and neuropathy) and reversed when the models included diabetes duration rather than current age.

While earlier studies have assessed the effects of age at diabetes diagnosis on diabetes complications, to our knowledge, this is the first systematic review and meta-analysis exploring associations between age at diabetes diagnosis and subsequent outcomes. Interdependence of current age, age at diabetes diagnosis and diabetes duration precluded investigation of all three variables simultaneously; hence the use of models containing either age at diabetes diagnosis adjusted for current age or age at diabetes diagnosis adjusted for 







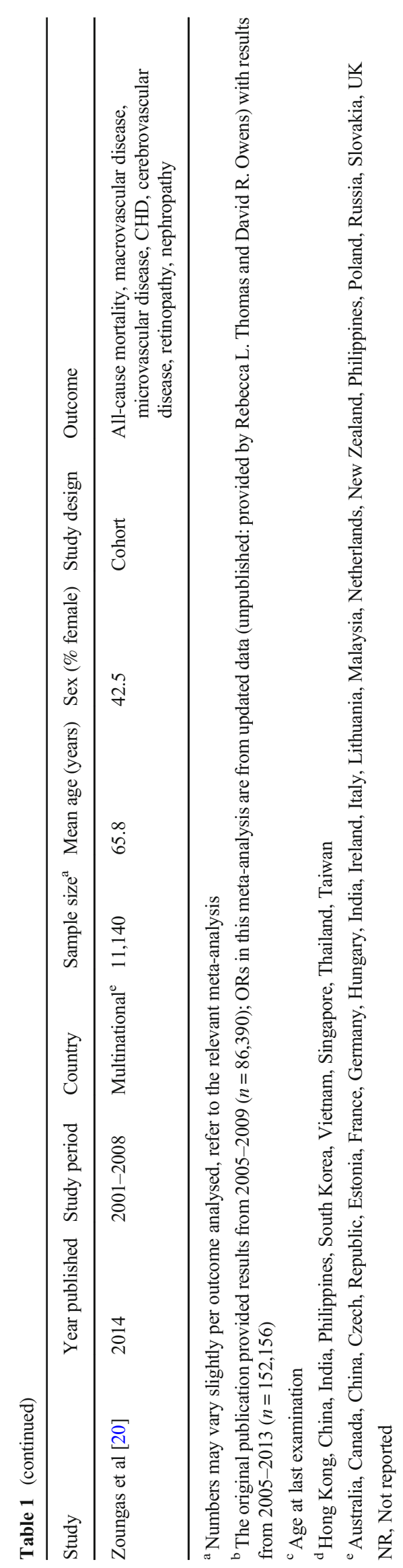


Fig. 2 Effect of age at diagnosis (per 1 year increase), adjusted for current age on the risk of all-cause mortality, macrovascular disease and microvascular disease. The size of the symbols is proportional to the study weight and horizontal lines represent $95 \%$ CIs

\author{
Outcome \\ All-cause mortality \\ Huo, L., et al [52] \\ Kenealy, T., et al [25] \\ Pavkov, M.E., et al [21] \\ Penno, G., et al [36] \\ Zoungas, S., et al [20] \\ Overall $\left(p<0.001, l^{2}=98 \%\right)$
}
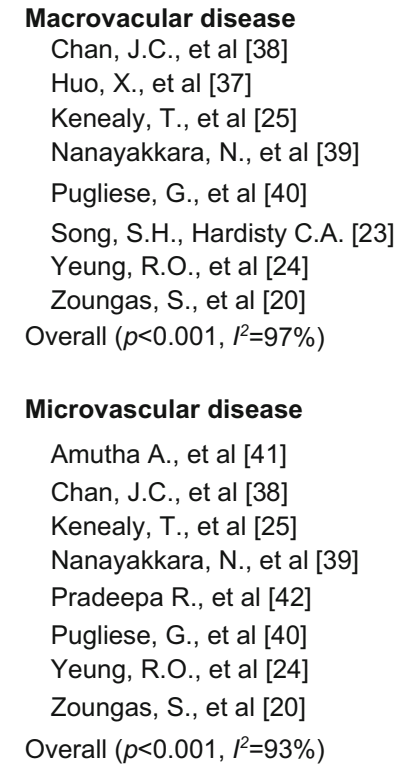

0.8
OR $(95 \% \mathrm{Cl})$

$0.95(0.95,0.96) \quad 744,188$

$0.98(0.97,0.99) \quad 70,057$

$0.90(0.89,0.92) \quad 2963$

$0.99(0.99,0.99) \quad 15,733$

$0.97(0.96,0.98) \quad 11,140$

$0.96(0.94,0.99) \quad 844,081$

$0.92(0.91,0.93) \quad 9506$

$0.97(0.97,0.97) \quad 222,770$

$0.97(0.97,0.98) \quad 48,444$

$0.97(0.96,0.98) \quad 3029$

$0.99(0.98,0.99) \quad 15,933$

$0.98(0.97,0.99) \quad 2,733$

$0.97(0.97,0.97) \quad 42,453$

$0.97(0.96,0.98) \quad 11,140$

$0.97(0.96,0.98) \quad 356,008$

$12(0.98,1.27) \quad 90$

$0.98(0.97,0.99) \quad 9506$

$0.96(0.95,0.96) \quad 65,547$

$0.93(0.92,0.93) \quad 3033$

$0.91(0.88,0.95) \quad 1608$

$0.96(0.95,0.96) \quad 15,733$

$0.95(0.95,0.96) \quad 42,453$

$0.95(0.94,0.96) \quad 11,140$

$0.95(0.94,0.96) \quad 149,110$ diabetes duration. People diagnosed with diabetes at an older age may be more likely to have accumulated adverse cardiovascular risk factors compared with those diagnosed at a younger age. Since advancing age is a powerful predictor of vascular complications, for the same diabetes duration, people diagnosed at a younger age are likely to have lower absolute risks of events as compared with people diagnosed at an older age. Over time, however, the effects of both ageing and disease duration may be amplified, resulting in premature complications and death in people diagnosed with type 2 diabetes at a younger age. To illustrate, a person diagnosed with type 2 diabetes at age 30 years would have a lower absolute risk of complications compared with a person diagnosed at age 50 years but by the time they both reach age 60 years the person diagnosed at a younger age would have a higher relative and absolute risk due to the effects of ageing, compounded by the effects of longer diabetes duration. This pattern has been observed in several young-onset type 2 diabetes populations $[21,55]$. Thus, younger people pose a significant challenge for clinicians and decision makers who need to be aware of these compounding pathologies of natural ageing and premature vascular ageing associated with type 2 diabetes. Further, people diagnosed with type 2 diabetes at a younger age still have the potential to develop complications at an earlier stage of life, at a time when the complications are more likely to cause greater disability and loss of productivity compared with people diagnosed at an older age.

There is a lack of RCT studies on achieving good glycaemic control and optimisation of cardiovascular risk factors in young-onset type 2 diabetes; many trials recruited middle-aged people with long disease duration at greatest absolute risk of complications. However, data from these older populations may not reflect the pathophysiology of type 2 diabetes in younger people, given evidence suggesting that the development of diabetes complications may differ between younger and older individuals. Further, many of these studies lack sufficient follow-up to capture complications in younger people who may have a longer time to event. The observations of this study and others examining type 2 diabetes complications $[15,23,38,51]$ add impetus to conducting trials examining this young cohort. There is an urgent need for data specifically pertaining to younger type 2 diabetes populations examining the trajectory of vascular complications and the impact of interventions (pharmacological as well as non-pharmacological approaches) to improve outcomes.

We found that age at diabetes diagnosis adjusted for current age was inversely associated with risk of all-cause death, 
Outcome

Nephropathy

Amutha A., et al [41]

Amutha, A., et al [43]

Kenealy, T., et al [25]

Nanayakkara, N., et al [39]

Pavkov, M.E., et al [21]

Romero-Aroca, P., et al [48]

Unnikrishnan, R, Anjana RM., et al [51] Older onset*

Unnikrishnan, R, Anjana RM., et al [51] Younger onset ${ }^{\star}$

Unnikrishnan, R, Rema M., et al [52]

Zoungas, S., et al [20]

Overall $(p<0.001, R=92 \%)$

\section{Neuropathy}

Amutha A., et al [41]

Amutha, A., et al [43]

Kenealy, T., et al [25]

Nanayakkara, N., et al [39]

Pradeepa R, Rema M., et al [45]

Song, S.H. and C.A. Hardisty [23]

Unnikrishnan, R, Anjana RM., et al [51] Older onset*

Unnikrishnan, R, Anjana RM., et al [51] Younger onset*

Overall $(p<0.001, R=48 \%)$

\section{Retinopathy}

Amutha A., et al [41]

Amutha, A., et al [43]

Cai, X., et al. [14]

Chen, M.S., et al 53]

Huo, X., et al [37]

Kenealy, T., et al [25]

Nanayakkara, N., et al [39]

Pugliese, G., et al [40]

Rema, M., et al [46]

Romero-Aroca, P., et al [48]

Song, S.H. and T.A. Gray [49]

Thomas, R.L et al [49]

Unnikrishnan, R, Anjana RM., et al [50] Older onset*

Unnikrishnan, R, Anjana RM., et al [50] Younger onset*

Wong, J., et al [15]

Zoungas, S., et al [20]

Overall $(p<0.001, R=97 \%)$

\section{Coronary heart disease}

Kenealy, T., et al [25]

Nanayakkara, N., et al [39]

Pugliese, G., et al [40]

Song, S.H. and C.A. Hardisty [23]

Zoungas, S., et al [20]

Overall $(p<0.001, R=54 \%)$

Cerebrovascular disease

Kenealy, T., et al [25]

Nanayakkara, N., et al [39]

Pugliese, G., et al [40]

Song, S.H. and C.A. Hardisty [23]

Zoungas, S., et al [20]

Overall $(p<0.001, R=75 \%)$

Peripheral vascular disease

Amutha A., et al [41]

Kenealy, T., et al [25]

Nanayakkara, N., et al [39]

Pradeepa R, Chella S., et al [44]

Pugliese, G., et al [40]

Song, S.H. and C.A. Hardisty [23]

Unnikrishnan, R, Anjana RM., et al [50] Older onset*

Unnikrishnan, R, Anjana RM., et al [50] Younger onset*

Zoungas, S., et al [20]

Overall $(p<0.001, R=82 \%)$

OR $(95 \% \mathrm{Cl})$

n

$1.04[0.88,1.22]$

$0.89[0.83,0.96]$

$0.96[0.95,0.96]$

$0.95[0.94,0.97]$

$0.90[0.88,0.91]$

$0.90[0.89,0.92]$

$0.95[0.85,1.06]$

0.97 [0.87, 1.08]

$0.97[0.95,0.99]$

$0.96[0.95,0.97]$

$0.94[0.92,0.96]$

90

840

66,128

2970

2726

15,030

267

173

267

11,140

$0.87[0.71,1.07]$

$0.91[0.83,1.00]$

$0.96[0.95,0.96]$

$0.95[0.94,0.96]$

0.97 [0.95, 0.99]

$0.94[0.93,0.95]$

$1.05[0.84,1.31]$

$1.05[0.84,1.31]$

$0.95[0.94,0.96]$

90

672

65,906

3358

1629

2733

256

240

74,884

$0.87[0.71,1.07]$

$0.91[0.85,0.98]$

$0.90[0.88,0.91]$

$0.99[0.89,1.10]$

$0.91[0.86,0.95]$

$0.91[0.86,0.96]$

$0.92[0.91,0.93]$

$1.01[1.00,1.03]$

$0.90[0.88,0.92]$

$0.91[0.89,0.94]$

$0.86[0.75,0.98]$

$0.90[0.90,0.90]$

$0.89[0.83,0.95]$

$0.86[0.75,0.98]$

$0.94[0.93,0.95]$

$0.97[0.96,0.98]$

$0.92[0.90,0.95]$

222,770

67,739

3261

15,933

1715

15,030

2516

152,156

240

177

1476

11,140

498,613

$0.97[0.96,0.98] \quad 59,228$

$0.98[0.97,0.99] \quad 3037$

$0.98[0.97,0.98] \quad 15,733$

$0.99[0.98,1.01] \quad 2733$

$0.98[0.97,0.99] \quad 11,140$

$0.98[0.97,0.98] \quad 91,871$

$0.98[0.97,0.99]$

$0.98[0.97,1.00]$

$0.96[0.96,0.97]$

$0.97[0.95,0.99]$

$0.98[0.97,0.99]$

$0.98[0.97,0.99]$

66,292

2982

15,933

2733

11,140

99,080

$2.26[0.12,42.25] \quad 672$

$0.96[0.95,0.98] \quad 65,906$

$0.97[0.95,0.98] \quad 3353$

$1.02[0.99,1.05] \quad 1755$

$0.96[0.95,0.96] \quad 15,733$

$0.95[0.93,0.97] \quad 2733$

$0.99[0.89,1.10] \quad 258$

$1.19[0.70,2.04] \quad 244$

$0.99[0.98,1.00] \quad 11,140$

$0.97[0.96,0.99] \quad 101,794$

0.25

Fig. 3 Effect of age at diagnosis (per 1 year increase), adjusted for current age on the risk of secondary outcomes. The symbols are proportional to the study weight and horizontal lines represent 95\% CIs. For
Unnikrishnan, R, Anjana RM., et al [50], older onset refers to those diagnosed aged $>50$ years and younger onset refers to those diagnosed aged $\leq 25$ years macrovascular disease and microvascular disease. Our findings underscore the importance of cardiovascular risk management among people with diabetes. Screening for and prevention of macrovascular complications is particularly important for older people with diabetes, who have the highest short-term absolute risk. Increasing age remains one of the 
most important risk factors for the development of macrovascular complications. However, it is also important to note that people diagnosed with diabetes at a younger age have a longer lifetime risk of developing significant complications, thus achieving good glycaemic control and optimisation of cardiovascular risk factors is of particular importance across their lifespan. This difference in risk between younger and older people in terms of absolute vs lifetime risks of type 2 diabetes complications, should perhaps be recognised in diabetes management guidelines, with increased promotion of screening programmes in older people with type 2 diabetes and a greater emphasis on preventive measures for younger people with type 2 diabetes.

As early intensive multifactorial risk factor intervention is important for the prevention of long-term macrovascular complications among people with newly diagnosed diabetes [56], our findings further suggest that this should be sustained long-term to minimise risks over time. Clearly, strategies are needed to ensure sustained adherence to lifestyle behaviours and therapies proven to have cardiovascular benefits among people with diabetes. Existing treatment guidelines are limited by being reactive to suboptimal glycaemic control after it has developed, without means to predict which people require intensified treatment. Refined stratification using age at diagnosis may provide a method of identifying, at diagnosis, those at greatest risk of complications who would most benefit from targeted, individualised treatment regimens. Moreover, public health measures to delay and/or prevent the onset of type 2 diabetes until older age may yield benefits by reducing the duration of diabetes and the burden of complications.

The development and progression of type 2 diabetes represents a complex interplay between genetic, epigenetic, lifestyle, demographic, socioeconomic, therapeutic and environmental factors. Given the myriad factors involved, and the variable reporting across included studies, it was difficult to establish uniformity in study definitions and covariate adjustment across studies. There was considerable variation in the definitions of 'younger' and 'older' age at type 2 diabetes diagnosis, with some studies defining 'younger' as $>30$ years of age, $>40$ years of age or $>50$ years of age. To mitigate this, we examined the effect of age at diabetes diagnosis (adjusted for current age), in yearly increments. Studies varied greatly with respect to measured confounding factors such as ethnicity, study country and year, type 2 diabetes diagnostic criteria, medication use, glycaemic control age, obesity, cardiometabolic risk factors, comorbid conditions, recruitment, source of participants, family history, healthcare access and sociodemographic factors. We were unable to adjust for these factors, as the data were either unavailable or not comparable due to the lack of standardised definitions across published studies. Moving forward, standardised approaches to reporting and complete data capture of relevant variables will assist with pooling and analysis of disparate datasets. This may be facilitated by the creation of international data registries. Performance bias (a potential difference in the care provided between early- and later-onset type 2 diabetes groups and between different centres) could not be assessed. Older people with type 2 diabetes may have cognitive impairment or other comorbidities precluding treatment intensification or even leading to de-intensification. Alternatively, people diagnosed with type 2 diabetes at a younger age may have been treated more intensively than people diagnosed at an older age. If this were the case, this bias would ameliorate the differences between groups, such that our data may actually underestimate the true extent of the effect of younger age at type 2 diabetes diagnosis. However, this appears less likely as several studies suggest that younger people with type 2 diabetes have poorer glycaemic control, lower adherence to therapy and inferior self-care practices compared with older people $[57,58]$. In fact, the data suggest that younger people with type 2 diabetes receive suboptimal medical attention, potentially due in part to an absence of clinical guidelines targeted to younger people with type 2 diabetes and possibly the underestimation of risks of complications in these individuals [38].

The strength of this meta-analysis is the extensive and comprehensive literature search and focus on studies examining younger and older people with type 2 diabetes. Six databases were searched, a risk of bias appraisal performed, and reanalyses were undertaken, enabling inclusion of data from more than a million people with type 2 diabetes worldwide. Collaboration with other authors facilitated more homogeneous data definitions, data integration and meta-analysis. We found that there was high concordance between the different studies included in the meta-analysis, such that the directions of the effects were consistent, although the magnitude of effects and the CIs varied. This may be due to differences in study size, although contributions from genetic, ethnic and healthcare variations in study populations cannot be excluded. Nevertheless, the direction of the effects was consistent across the studies from different countries.

As with many systematic reviews and meta-analyses, this meta-analysis has some limitations. Not all identified studies were included in the meta-analysis due to difficulties sourcing comparable data from authors. The inability to acquire data from all eligible studies is not unexpected and is a part of the metaanalysis process [59]. We based our classification of age at type 2 diabetes diagnosis on the definitions used in each individual study, even though these definitions may have differed. It would be impossible to apply retrospectively a single definition of age at diagnosis to a large number of samples characterised with different variables in different studies. Additionally, the criteria for the diagnosis and classification of type 2 diabetes have changed with the advent of new technologies, such as the determination of pancreatic autoantibodies and C-peptide levels, as have the methods used to differentiate type 2 diabetes from other forms of diabetes (principally type 1 and monogenic diabetes). Lastly, 
due in part to the nature of the study question, the included studies were observational in design and therefore subject to the potential biases (confounding and selection) inherent to analyses of observational data. However, meta-analyses of observational studies can provide valuable insights, especially when RCTs are unavailable or are inappropriate for addressing the question [34], as is the case here. Findings from this review are based on observational data and therefore causality may not be attributed. Thus, although these findings may be applicable on a population level, any recommendations need to be individualised to the clinical situation of each person with type 2 diabetes.

We have completed the first systematic review and metaanalysis examining the effects of age at type 2 diabetes diagnosis on all-cause mortality, microvascular complications and macrovascular complications. This comprehensive analysis, comprising over a million participants, indicates that when adjusted for current age, younger age at type 2 diabetes diagnosis is associated with increased risk of mortality, macrovascular complications and macrovascular complications. Identification and quantification of the increased risk of mortality and vascular disease conferred by younger age at type 2 diabetes diagnosis may enable risk stratification of people early in the condition and thereby provide greater opportunities for interventions to reduce risk of complication-associated morbidity and mortality for this increasing population demographic developing type 2 diabetes.

Supplementary Information The online version contains supplementary material available at https://doi.org/10.1007/s00125-020-05319-w.

Acknowledgements We would like to acknowledge the assistance of M. Misso (Monash Centre for Health Research and Implementation, Monash University, Clayton, VIC, Australia) and E. Lilburn (School Public Health and Preventive Medicine, Monash University, Melbourne, VIC, Australia - retired) in completing this work.

Data availability Application for datasets generated during and/or analysed during the current study may be considered by the corresponding author on reasonable request.

Funding HJT and SZ are NHMRC research fellows. This research has received no specific grant from any other funding agency in the public, commercial or not-for profit sectors. The authors had full responsibility for data collection, data interpretation and writing of the report. The funding source had no role in the study design, data collection, data analysis, data interpretation, writing of the report or the decision to submit for publication.

Disclaimer The findings and conclusions in this report are those of the authors and do not necessarily represent the official position of the Centers for Disease Control and Prevention.

Authors' relationships and activities DRO reports receiving honoraria from Boehringer Ingelheim, Eli Lilly, Novo Nordisk, Mannheim, Roche Diagnostics and Sanofi for research, travel and advisory panel activities. JW, independently and on behalf of institutions with which she is associated, has received research funds, travel grants and speaker/advisory honoraria from various companies including Eli Lilly and Company, Boehringer lngelheim, Novo Nordisk, Merck, AstraZeneca, BristolMeyers Squibb, Novartis, Sanofi and Servier. SS reports receiving lecture fees from Novo Nordisk, Takeda and Astra Zeneca. GP has received travel grants and speaker/advisory honoraria from AstraZeneca, Boehringer Ingelheim, Eli Lilly Italia, Merck Sharp \& Dohme, Novo Nordisk and Takeda Italia. JC reports receiving research grants from Servier for the ADVANCE trial and post-trial study and honoraria for speaking about these studies at Scientific meetings. SZ reports the following disclosures outside the submitted work: for AstraZeneca, participation in advisory board, expert committees or educational meetings in 2017 and 2018 on behalf of Monash University with payment to institution; for NovoNordisk, participation in advisory board, expert committees or educational meetings in 2016 and 2018 on behalf of Monash University with payment to institution; for Sanofi, participation in advisory board, expert committees or educational meetings in 2018 on behalf of Monash University with payment to institution; for Boehringer Ingelheim, participation in advisory board, expert committees or educational meetings in 2019 on behalf of Monash University with payment to institution. All other authors declare that there are no relationships or activities that might bias, or be perceived to bias, their work.

Contribution statement NN, AMG, HJT, AJC and SZ were responsible for the concept and design of the study. AJC, SH, HJT, and SZ supervised the study. NN, AJC, SH, AMG, MEP, GP, TK, DRO, RLT, JW, SS, JCNC, AOYL, LJ, DJM, VM, AA, DG, PRA, JC and SZ contributed to data acquisition, analysis or interpretation. NN, AMG and SH carried out statistical analysis. The manuscript was drafted by NN, AJC, SH, HJT and SZ. All authors revised the manuscript critically and approved the final version. NN, AMG, SH and SZ had full access to the data and take responsibility for the integrity of the data and accuracy of the analysis.

Open Access This article is licensed under a Creative Commons Attribution 4.0 International License, which permits use, sharing, adaptation, distribution and reproduction in any medium or format, as long as you give appropriate credit to the original author(s) and the source, provide a link to the Creative Commons licence, and indicate if changes were made. The images or other third party material in this article are included in the article's Creative Commons licence, unless indicated otherwise in a credit line to the material. If material is not included in the article's Creative Commons licence and your intended use is not permitted by statutory regulation or exceeds the permitted use, you will need to obtain permission directly from the copyright holder. To view a copy of this licence, visit http://creativecommons.org/licenses/by/4.0/.

\section{References}

1. Rahelic D (2016) 7th edition of IDF diabetes atlas: call for immediate action. Lijec Vjesn 138:57-58 [article in Croatian]

2. Song SH, Hardisty CA (2008) Early-onset type 2 diabetes mellitus: an increasing phenomenon of elevated cardiovascular risk. Expert Rev Cardiovasc Ther 6:315-322

3. Sullivan MD, Anderson RT, Aron D et al (2007) Health-related quality of life and cost-effectiveness components of the Action to Control Cardiovascular Risk in Diabetes (ACCORD) trial: rationale and design. Am J Cardiol 99:90i-102i

4. Barr EL, Zimmet PZ, Welborn TA et al (2007) Risk of cardiovascular and all-cause mortality in individuals with diabetes mellitus, impaired fasting glucose, and impaired glucose tolerance: the Australian Diabetes, Obesity, and Lifestyle Study (AusDiab). 
Circulation 116:151-157. https://doi.org/10.1161/ CIRCULATIONAHA.106.685628

5. Gungor N, Bacha F, Saad R, Janosky J, Arslanian S (2005) Youth type 2 diabetes: insulin resistance, beta-cell failure, or both? Diabetes Care 28:638-644. https://doi.org/10.2337/diacare.28.3. 638

6. Gungor N, Thompson T, Sutton-Tyrrell K, Janosky J, Arslanian S (2005) Early signs of cardiovascular disease in youth with obesity and type 2 diabetes. Diabetes Care 28:1219-1221. https://doi.org/ 10.2337/diacare.28.5.1219

7. Song SH (2016) Early-onset type 2 diabetes: high lifetime risk for cardiovascular disease. Lancet Diabetes Endocrinol 4:87-88. https://doi.org/10.1016/S2213-8587(15)00390-3

8. Gungor N, Hannon T, Libman I, Bacha F, Arslanian S (2005) Type 2 diabetes mellitus in youth: the complete picture to date. Pediatr Clin N Am 52:1579-1609. https://doi.org/10.1016/j.pcl.2005.07. 009

9. Zou X, Zhou X, Ji L et al (2017) The characteristics of newly diagnosed adult early-onset diabetes: a population-based crosssectional study. Sci Rep 7:46534

10. Ahlqvist E, Storm P, Karajamaki A et al (2018) Novel subgroups of adult-onset diabetes and their association with outcomes: a datadriven cluster analysis of six variables. Lancet Diabetes Endocrinol 6:361-369. https://doi.org/10.1016/S2213-8587(18) 30051-2

11. Hillier TA, Pedula KL (2003) Complications in young adults with early-onset type 2 diabetes: losing the relative protection of youth. Diabetes Care 26:2999-3005. https://doi.org/10.2337/diacare.26. 11.2999

12. Luk AO, Lau ES, So WY et al (2014) Prospective study on the incidences of cardiovascular-renal complications in Chinese patients with young-onset type 1 and type 2 diabetes. Diabetes Care 37:149-157

13. Xu H, Aldrich MC, Chen $\mathrm{Q}$ et al (2015) Validating drug repurposing signals using electronic health records: a case study of metformin associated with reduced cancer mortality. J Am Med Inform Assoc 22:179-191

14. Cai X, Han X, Zhang S, Luo Y, Chen Y, Ji L (2014) Age at diagnosis and C-peptide level are associated with diabetic retinopathy in Chinese. PLoS One 9:e91174. https://doi.org/10.1371/ journal.pone.0091174

15. Wong J, Molyneaux L, Constantino M, Twigg SM, Yue DK (2008) Timing is everything: age of onset influences long-term retinopathy risk in type 2 diabetes, independent of traditional risk factors. Diabetes Care 31:1985-1990. https://doi.org/10.2337/dc08-0580

16. Ohno T, Kato N, Shimizu M et al (1993) Effect of age on the development or progression of albuminuria in non-insulindependent diabetes mellitus (NIDDM) without hypertension. Diabetes Res 22:115-121

17. Klein R, Klein BE, Moss SE, Cruickshanks KJ (1995) Ten-year incidence of gross proteinuria in people with diabetes. Diabetes 44 : 916-923. https://doi.org/10.2337/diab.44.8.916

18. Mohan V, Vijayaprabha R, Rema M (1996) Vascular complications in long-term south Indian NIDDM of over 25 years duration. Diabetes Res Clin Pract 31:133-140. https://doi.org/10.1016/ 0168-8227(96)01215-6

19. Davis TM, Stratton IM, Fox CJ, Holman RR, Turner RC (1997) U.K. Prospective Diabetes Study 22. Effect of age at diagnosis on diabetic tissue damage during the first 6 years of NIDDM. Diabetes Care 20:1435-1441. https://doi.org/10.2337/diacare.20.9.1435

20. Zoungas S, Woodward M, Li Q et al (2014) Impact of age, age at diagnosis and duration of diabetes on the risk of macrovascular and microvascular complications and death in type 2 diabetes. Diabetologia 57:2465-2474. https://doi.org/10.1007/s00125-0143369-7
21. Pavkov ME, Bennett PH, Knowler WC, Krakoff J, Sievers ML, Nelson RG (2006) Effect of youth-onset type 2 diabetes mellitus on incidence of end-stage renal disease and mortality in young and middle-aged Pima Indians. JAMA 296:421-426. https://doi.org/10. 1001/jama.296.4.421

22. Yeap BB, McCaul KA, Flicker L et al (2015) Diabetes, myocardial infarction and stroke are distinct and duration-dependent predictors of subsequent cardiovascular events and all-cause mortality in older men. J Clin Endocrinol Metab 100:1038-1047. https://doi.org/10. 1210/jc.2014-3339

23. Song SH, Hardisty CA (2009) Early onset type 2 diabetes mellitus: a harbinger for complications in later years: clinical observation from a secondary care cohort. QJM 102:799-806. https://doi.org/ 10.1093/qjmed/hcp121

24. Yeung RO, Zhang Y, Luk A et al (2014) Metabolic profiles and treatment gaps in young-onset type 2 diabetes in Asia (the JADE programme): a cross-sectional study of a prospective cohort. Lancet Diabetes Endocrinol 2:935-943. https://doi.org/10.1016/S22138587(14)70137-8

25. Kenealy T, Elley CR, Robinson E et al (2008) An association between ethnicity and cardiovascular outcomes for people with type 2 diabetes in New Zealand. Diabet Med 25:1302-1308. https://doi.org/10.1111/j.1464-5491.2008.02593.x

26. Stroup DF, Berlin JA, Morton SC et al (2000) Meta-analysis of observational studies in epidemiology: a proposal for reporting. Meta-analysis Of Observational Studies in Epidemiology (MOOSE) group. Jama 283:2008-2012

27. Moher D, Liberati A, Tetzlaff J, Altman DG, Group P (2009) Preferred reporting items for systematic reviews and meta-analyses: the PRISMA Statement. Open Med 3:e123-e130

28. (Monash University and Monash Health M, Australia (2014) Monash Centre for Health Research and Implementation (MCHRI) Evidence Synthesis Program template for critical appraisal of a cohort study. In: (adapted from Critical Appraisal Templates (2010) Centre for Clinical Effectiveness SH, Melbourne, Australia (ed)

29. Wells G, Shea B, O'Connell D, et al (2000) The Newcastle-Ottawa Scale (NOS) for assessing the quality of nonrandomized studies in meta-analyses. Available from http://www.ohri.ca/programs/ clinical_epidemiology/oxford.asp. Accessed 28 Oct 2020

30. The Nordic Cochrane Centre (2014) Review Manager (RevMan). The Cochrane Collaboration, Copenhagen

31. DerSimonian R, Laird N (1986) Meta-analysis in clinical trials. Control Clin Trials 7:177-188. https://doi.org/10.1016/01972456(86)90046-2

32. Senn SS (2008) Statistical issues in drug development. John Wiley \& Sons

33. Higgins JP, Thompson SG (2002) Quantifying heterogeneity in a meta-analysis. Stat Med 21:1539-1558. https://doi.org/10.1002/ sim. 1186

34. Egger M, Schneider M, Davey Smith G (1998) Spurious precision? Meta-analysis of observational studies. BMJ 316:140-144. https:// doi.org/10.1136/bmj.316.7125.140

35. Egger M, Davey Smith G, Schneider M, Minder C (1997) Bias in meta-analysis detected by a simple, graphical test. BMJ 315:629634. https://doi.org/10.1136/bmj.315.7109.629

36. Penno G, Solini A, Orsi E et al (2018) Non-albuminuric renal impairment is a strong predictor of mortality in individuals with type 2 diabetes: the Renal Insufficiency And Cardiovascular Events (RIACE) Italian multicentre study. Diabetologia 61:22772289. https://doi.org/10.1007/s00125-018-4691-2

37. Huo X, Gao L, Guo L et al (2016) Risk of non-fatal cardiovascular diseases in early-onset versus late-onset type 2 diabetes in China: a cross-sectional study. Lancet Diabetes Endocrinol 4:115-124. https://doi.org/10.1016/S2213-8587(15)00508-2 
38. Chan JC, Lau ES, Luk AO et al (2014) Premature mortality and comorbidities in young-onset diabetes: a 7-year prospective analysis. Am J Med 127:616-624. https://doi.org/10.1016/j.amjmed. 2014.03.018

39. Nanayakkara N, Ranasinha S, Gadowski A et al (2018) Age, age at diagnosis and diabetes duration are all associated with vascular complications in type 2 diabetes. J Diabetes Complicat 32:279290. https://doi.org/10.1016/j.jdiacomp.2017.11.009

40. Pugliese G, Solini A, Zoppini G et al (2012) High prevalence of advanced retinopathy in patients with type 2 diabetes from the Renal Insufficiency And Cardiovascular Events (RIACE) Italian Multicenter Study. Diabetes Res Clin Pract 98:329-337. https:// doi.org/10.1016/j.diabres.2012.09.006

41. Amutha A, Anjana RM, Venkatesan U et al (2017) Incidence of complications in young-onset diabetes: comparing type 2 with type 1 (the young diab study). Diabetes Res Clin Pract 123:1-8. https:// doi.org/10.1016/j.diabres.2016.11.006

42. Pradeepa R, Anjana RM, Unnikrishnan R, Ganesan A, Mohan V, Rema M (2010) Risk factors for microvascular complications of diabetes among South Indian subjects with type 2 diabetes: the Chennai Urban Rural Epidemiology Study (CURES) Eye Study5. Diabetes Technol Ther 12:755-761. https://doi.org/10.1089/dia. 2010.0069

43. Amutha A, Datta M, Unnikrishnan IR et al (2011) Clinical profile of diabetes in the young seen between 1992 and 2009 at a specialist diabetes centre in south India. Prim Care Diabetes 5:223-229. https://doi.org/10.1016/j.pcd.2011.04.003

44. Pradeepa R, Chella S, Surendar J, Indulekha K, Anjana RM, Mohan V (2014) Prevalence of peripheral vascular disease and its association with carotid intima-media thickness and arterial stiffness in type 2 diabetes: the Chennai urban rural epidemiology study (CURES 111). Diab Vasc Dis Res 11:190-200. https://doi.org/10. 1177/1479164114524584

45. Pradeepa R, Rema M, Vignesh J, Deepa M, Deepa R, Mohan V (2008) Prevalence and risk factors for diabetic neuropathy in an urban south Indian population: the Chennai Urban Rural Epidemiology Study (CURES-55). Diabet Med 25:407-412. https://doi.org/10.1111/j.1464-5491.2008.02397.x

46. Rema M, Premkumar S, Anitha B, Deepa R, Pradeepa R, Mohan V (2005) Prevalence of diabetic retinopathy in urban India: the Chennai Urban Rural Epidemiology Study (CURES) eye study, I. Invest Ophthalmol Vis Sci 46:2328-2333. https://doi.org/10.1167/iovs.050019

47. Romero-Aroca P, Navarro-Gil R, Valls-Mateu A, Sagarra-Alamo R, Moreno-Ribas A, Soler N (2017) Differences in incidence of diabetic retinopathy between type 1 and 2 diabetes mellitus: a nine-year follow-up study. Br J Ophthalmol 101:1346-1351. https://doi.org/10.1136/bjophthalmol-2016-310063
48. Song SH, Gray TA (2011) Early-onset type 2 diabetes: high risk for premature diabetic retinopathy. Diabetes Res Clin Pract 94:207211. https://doi.org/10.1016/j.diabres.2011.07.030

49. Thomas RL, Dunstan FD, Luzio SD et al (2015) Prevalence of diabetic retinopathy within a national diabetic retinopathy screening service. Br J Ophthalmol 99:64-68

50. Unnikrishnan R, Anjana RM, Amutha A et al (2017) Youngeronset versus older-onset type 2 diabetes: clinical profile and complications. J Diabetes Complicat 31:971-975. https://doi.org/10.1016/ j.jdiacomp.2017.03.007

51. Unnikrishnan RI, Rema M, Pradeepa R et al (2007) Prevalence and risk factors of diabetic nephropathy in an urban South Indian population: the Chennai Urban Rural Epidemiology Study (CURES 45). Diabetes Care 30:2019-2024. https://doi.org/10.2337/dc06-2554

52. Huo L, Magliano DJ, Ranciere F et al (2018) Impact of age at diagnosis and duration of type 2 diabetes on mortality in Australia 1997-2011. Diabetologia 61:1055-1063. https://doi.org/10.1007/ s00125-018-4544-z

53. Chen MS, Kao CS, Chang CJ et al (1992) Prevalence and risk factors of diabetic retinopathy among noninsulin-dependent diabetic subjects. Am J Ophthalmol 114:723-730. https://doi.org/10. 1016/S0002-9394(14)74051-6

54. Hamman RF, Mayer EJ, Moo-Young GA, Hildebrandt W, Marshall JA, Baxter J (1989) Prevalence and risk factors of diabetic retinopathy in non-Hispanic whites and Hispanics with NIDDM. San Luis Valley Diabetes Study. Diabetes 38:1231-1237. https:// doi.org/10.2337/diab.38.10.1231

55. Yokoyama H, Okudaira M, Otani T et al (2000) Higher incidence of diabetic nephropathy in type 2 than in type 1 diabetes in earlyonset diabetes in Japan. Kidney Int 58:302-311. https://doi.org/10. 1046/j.1523-1755.2000.00166.x

56. Holman RR, Paul SK, Bethel MA, Matthews DR, Neil HA (2008) 10-year follow-up of intensive glucose control in type 2 diabetes. $\mathrm{N}$ Engl J Med 359:1577-1589. https://doi.org/10.1056/ NEJMoa0806470

57. TODAY Study Group, Zeitler P, Hirst K et al (2012) A clinical trial to maintain glycemic control in youth with type 2 diabetes. $\mathrm{N}$ Engl $\mathrm{J}$ Med 366:2247-2256. https://doi.org/10.1056/NEJMoa1109333

58. Nanayakkara N, Pease AJ, Ranasinha S et al (2018) Younger people with type 2 diabetes have poorer self-care practices compared with older people: results from the Australian National Diabetes Audit. Diabet Med 35:1087-1095. https://doi.org/10. 1111/dme.13660

59. Kelley GA, Kelley KS, Tran ZV (2004) Retrieval of missing data for meta-analysis: a practical example. Int J Technol Assess Health Care 20:296-299. https://doi.org/10.1017/S0266462304001114

Publisher's note Springer Nature remains neutral with regard to jurisdictional claims in published maps and institutional affiliations. 